\title{
Pembrolizumab-Induced Immune- Mediated Colitis in a Patient with Concurrent Clostridium Difficile Infection
}

\author{
Cheng Zhou $^{a} \quad$ Yael Klionsky $^{b} \quad$ Michelle E. Treasure $^{c} \quad$ Debora S. Bruno $^{d}$ \\ aDepartment of Medicine-Pediatrics, MetroHealth Medical Center, Cleveland, OH, USA; \\ ${ }^{b}$ Department of Internal Medicine, MetroHealth Medical Center, Cleveland, $\mathrm{OH}$, USA; \\ 'Department of Internal Medicine, Hematology and Oncology Division, Cleveland Clinic \\ Foundation, Cleveland, OH, USA; dDepartment of Internal Medicine, Hematology and \\ Oncology Division, University Hospitals - Seidman Cancer Center, Cleveland, OH, USA
}

\section{Keywords}

Pembrolizumab · Immune-mediated colitis · Immune-related adverse events (irAEs) ·

Clostridium difficile infection (CDI)

\begin{abstract}
Pembrolizumab is a programmed death receptor-1 (PD-1) inhibitor that has been approved for treatment of a wide variety of malignancies. Immune-mediated colitis is a known but uncommon adverse effect of pembrolizumab. Symptoms of immune-mediated colitis can be similar to those of many other gastrointestinal illnesses, including Clostridium difficile infection (CDI). If not recognized and treated in a timely fashion, immune-mediated colitis can lead to significant morbidity in cancer patients. We report the case of a 56-year-old woman on pembrolizumab for metastatic non-small cell lung cancer (NSCLC) who presented with severe colitis symptoms and initially tested positive for $\mathrm{CDI}$. Her colitis symptoms worsened despite appropriate treatment for $\mathrm{CDI}$ but later improved rapidly after systemic corticosteroid was started
\end{abstract}




\section{Case Reports in Oncology}

Case Rep Oncol 2019;12:164-170

DOI: $10.1159 / 000497155$ (C) 2019 The Author(s).
www.karger.com/cro

2019 The Author(s). Published by S. Karger AG, Basel

Zhou et al.: Pembrolizumab-Induced Immune-Mediated Colitis in a Patient with Concurrent Clostridium Difficile Infection

for suspected immune-mediated colitis. To our knowledge, this is the first reported case of concurrent pembrolizumab-induced colitis and CDI. Immune-mediated colitis should be considered in the differential diagnoses in patients on pembrolizumab or other immune checkpoint inhibitors who present with colitis symptoms, even when a concurrent infectious etiology is suspected.

(C) 2019 The Author(s)

Published by S. Karger AG, Basel

\section{Introduction}

Pembrolizumab is a humanized monoclonal antibody blocking the PD-1 receptor. It has been approved for treatment of a wide variety of malignancies [1]. Immune-mediated colitis is a known adverse effect of pembrolizumab. Moderate to severe immune-mediated colitis affects $1 \%$ to $2 \%$ of patients receiving pembrolizumab $[2,3]$. Moderate to severe cases of pembrolizumab-induced colitis typically improve with systemic corticosteroid treatment [4]. Clostridium difficile infection (CDI) is a gastrointestinal infection that causes colitis and results in significant morbidity and mortality in cancer patients $[5,6]$. Symptoms of immune-mediated colitis and C. difficile colitis can be similar and occasionally concurrent, making the clinical picture more complicated.

We present the case of a 56-year-old woman on pembrolizumab for metastatic NSCLC who suffered from concurrent CDI and pembrolizumab-induced immune-mediated colitis. Her symptoms worsened despite aggressive CDI treatment but later improved rapidly and markedly when corticosteroid was started for immune-mediated colitis.

\section{Case Presentation}

A 56-year-old woman with metastatic NSCLC treated with pembrolizumab, pemetrexed and carboplatin was admitted to the hospital for dehydration and hypokalemia secondary to profuse, watery, intermittently bloody diarrhea for 10 days. She also complained of diffuse abdominal pain, poor oral intake and weight loss. Her cancer treatment had started 9 weeks prior to this admission with pembrolizumab, pemetrexed and carboplatin. Seven days prior to admission, she had received her third cycle of cancer therapy. On that day, she had complained of persistent diarrhea and had a positive C. difficile stool polymerase chain reaction (PCR) test. She was thus started on oral metronidazole for C. difficile treatment in the outpatient setting. The patient's symptoms worsened despite oral metronidazole, leading to hospitalization.

Upon hospital admission, the patient reported intermittent abdominal pain, stool urgency and diarrhea (4-6 episodes daily) that had not improved after five days of outpatient oral metronidazole therapy. Her weight had decreased by 5 pounds over the past seven days. She endorsed decreased oral intake but denied nausea and vomiting. Her vital signs were within normal limits. Laboratory tests on day of admission were significant for hypokalemia, hypochloremia, leukopenia, anemia and neutropenia (Table 1). She was started on intravenous metronidazole (500 mg every $8 \mathrm{~h}$ ) and oral vancomycin (initially $125 \mathrm{mg}$ every $6 \mathrm{~h}$ and a day later increased to $500 \mathrm{mg}$ every $6 \mathrm{~h}$ ) for treatment of severe CDI. She was also treated with 


\section{Case Reports in Oncology}

Case Rep Oncol 2019;12:164-170

DOI: $10.1159 / 000497155$

(c) 2019 The Author(s). Published by S. Karger AG, Basel www.karger.com/cro

Zhou et al.: Pembrolizumab-Induced Immune-Mediated Colitis in a Patient with Concurrent Clostridium Difficile Infection

potassium supplementation and intravenous fluids. Her symptoms worsened despite antibiotics. On hospital day 3, she had 10 episodes of diarrhea and constant diffuse abdominal pain. Abdominal CT (Fig. 1) showed pan-colitis and terminal ileitis without toxic megacolon. Given the lack of response to aggressive CDI treatment, pembrolizumab-induced immune-mediated colitis was suspected.

On hospital day 4, repeat stool C difficile PCR test was negative. Colonoscopy was not performed due to worsening neutropenia ( $60 \mathrm{cells} / \mathrm{mm}^{3}$ ). Intravenous methylprednisolone 1.5 $\mathrm{mg} / \mathrm{kg} /$ day was started. In response, patient showed rapid improvement, with less frequent and more formed stools (Fig. 2). Her abdominal pain resolved, and her oral intake markedly improved. On hospital day 7, she only had two soft bowel movements. On hospital day 9, she continued to have twice daily formed stools and was discharged home on an oral prednisone taper. Thirty days after discharge, she remained diarrhea-free and repeat abdominal CT showed complete resolution of the colitis and ileitis (Fig. 1).

\section{Discussion}

Pembrolizumab is approved for treatment of a wide and expanding variety of malignancies, such as NSCLC, melanoma, renal cell carcinoma, squamous cell carcinoma of the head and neck [1]. Pembrolizumab inhibits the PD-1: PD-L pathway by binding to the PD-1 receptor (expressed on T-cells, B-cells, macrophages and some dendritic cells), thus preventing the PD1 receptor from binding to its ligands, PD-L1 and PD-L2 [7]. In the normal human body, the PD-1: PD-L pathway is a negative co-stimulatory pathway that down-regulates T-cell activity. This pathway's most notable function is to foster self-tolerance and decrease autoimmunity. It achieves this function mainly by inducing anergy, deletion and decreased cytokine release in auto-reactive T-cells, as well as by pushing naïve T-cells towards developing into induced regulatory T-cells (iTregs) $[1,4,7,8]$. In the normal human body, PD-L1 is expressed broadly in hematopoietic cells and various peripheral tissues [7]. In some cancers, PD-L1 is expressed in tumor cells and tumor-infiltrating lymphocytes [1]. These cancers thus dampen the host's anti-cancer immune defense by binding to the host's PD-1 receptors and thereby co-opting the host's T-cells into tolerating the existence and expansion of malignancies. Pembrolizumab, by blocking the PD-1 receptor, disables PD-L1-expressing cancers from undermining the host's immune defense against them.

However, just as pembrolizumab liberates the host's immune defense against malignancies, it also unleashes the host's autoimmunity towards self-tissue and organ systems. Thus immune-related adverse events (irAEs) are a major side effect of pembrolizumab. A wide spectrum of irAEs have been reported, including pneumonitis, colitis, hepatitis, myositis, myocarditis, nephritis, myasthenia gravis and endocrinopathies [9, 10]. Immune-mediated colitis associated with pembrolizumab has an overall incidence of less than 4\% [9] and a reported incidence of $1.9 \%$ in NSCLC patients in particular [7]. Although pembrolizumab-induced immune-mediated colitis is rarely fatal [10], it can be associated with significant morbidity. Colonic perforation requiring emergent bowel resection has been reported in patients on pembrolizumab treatment who developed severe immune-mediated colitis [11].

Diarrhea is the most common clinical manifestation of immune-mediated colitis associated with pembrolizumab and other immune checkpoint inhibitors. The median interval 


\section{Case Reports in Oncology}

Case Rep Oncol 2019;12:164-170

DOI: $10.1159 / 000497155$

(c) 2019 The Author(s). Published by S. Karger AG, Base www.karger.com/cro

Zhou et al: Pembrolizumab-Induced Immune-Mediated Colitis in a Patient with Concurrent Clostridium Difficile Infection

between medication initiation and onset of diarrhea is 8 weeks (range: 1-32 weeks) [4]. Treatment strategies for immune-mediated colitis are largely determined by severity of diarrhea. Grade 1 and 2 diarrhea ( 7 or fewer episodes per day) can usually be managed with supportive therapy (hydration, diet modification, loperamide) [4] and oral budesonide [3]. Grade 3 and 4 diarrhea ( $>7$ episodes per day) is treated with systemic (intravenous followed by oral) corticosteroids, and the tumor necrosis factor alpha (TNF- $\alpha$ ) inhibitor infliximab is given in steroid-refractory cases $[3,4,11]$. Before initiating treatment for immune-mediated colitis, infectious causes of diarrhea should be ruled out.

The case we present in this report is unusual because immune-mediated colitis was concurrent with CDI. Specifically, it was the failure of CDI treatment that raised suspicion for immune-mediated colitis. This is the first reported case of concurrence of pembrolizumab-induced immune-mediated colitis and C. difficile colitis. To our knowledge, only one similar case has been reported to date by Gupta and Khanna in 2015. In their case, a 72-year-old male was receiving ipilimumab (a cytotoxic T-lymphocyte-associated protein 4 inhibitor) and nivolumab (a PD-1 inhibitor) for high-grade non-Hodgkin's lymphoma. He had diarrhea for 6 months and positive stool C diff PCR. His diarrhea did not improve with two 14-day courses of metronidazole and oral vancomycin followed by a long vancomycin taper. Immune-mediated colitis was suspected. Repeat $C$ diff testing was then performed and negative. This patient's diarrhea, like that of our patient's, resolved promptly with systemic corticosteroids [12]. In both our case and the case reported by Gupta and Khanna, concurrent CDI made the diagnosis of immune-mediated colitis very challenging. In both cases repeat $\mathrm{C}$. difficile testing was performed and negative just prior to starting corticosteroids, and in both cases systemic corticosteroids led to good outcome.

With the increasing use of pembrolizumab and other checkpoint inhibitors in cancer therapy, drug-induced, immune-mediated colitis and other irAEs are becoming an increasingly prominent clinical topic. Cancer patients are frequently exposed to multiple risk factors for CDI, such as chemotherapy, antibiotic use and frequent hospitalizations [5] and are susceptible to severe morbidity and mortality from CDI [6]. Therefore, it is highly likely that more cases of co-occurring immune-mediated colitis and CDI will be seen.

In addition to acknowledging and managing such concurrence, we also wonder whether CDI could trigger or exacerbate drug-induced, immune-mediated colitis. Further investigation has yet to be done in this particular topic. However, research has shown that history of CDI worsens inflammatory bowel disease (IBD) course over a 2-year period of time [13].

\section{Conclusions}

CDI and pembrolizumab-induced immune-mediated colitis can co-occur. In patients on pembrolizumab or other immune checkpoint inhibitors who present with colitis symptoms, immune-mediated colitis should be considered in the differential diagnoses even in the presence of a recent positive $\mathrm{C}$. difficile test, particularly if the patient's colitis symptoms fail to improve with appropriate CDI treatment. Repeat testing for C. difficile and other causes of infectious colitis should be performed prior to starting systemic steroids to treat immunemediated colitis. Future research should further investigate the interplay between CDI and immune-mediated colitis. 
Zhou et al.: Pembrolizumab-Induced Immune-Mediated Colitis in a Patient with Concurrent Clostridium Difficile Infection

\section{Statement of Ethics}

The authors have no ethical conflicts to disclose.

\section{Disclosure Statement}

The authors have no conflicts of interest to disclose.

\section{Author Contributions}

All authors have contributed in writing and reviewing the manuscript.

\section{References}

1 Meyers DE, Bryan PM, Banerji S, Morris DG. Targeting the PD-1/PD-L1 axis for the treatment of non-smallcell lung cancer. Curr Oncol. 2018 Aug;25(4):e324-34.

2 Pai-Scherf L, Blumenthal GM, Li H, Subramaniam S, Mishra-Kalyani PS, He K, et al. FDA approval summary: Pembrolizumab for treatment of metastatic non-small cell lung cancer: First-line therapy and beyond. Oncologist. 2017 Nov;22(11):1392-9.

3 Friedman CF, Proverbs-Singh TA, Postow MA. Treatment of the immune-related adverse effects of immune checkpoint inhibitors: A review. JAMA Oncol. 2016 Oct;2(10):1346-53.

4 Assarzadegan N, Montgomery E, Anders RA. Immune checkpoint inhibitor colitis: the flip side of the wonder drugs. Virchows Arch. 2018 Jan;472(1):125-33.

5 Chang GY, Dembry LM, Banach DB. Epidemiology of Clostridium difficile infection in hospitalized oncology patients. Am J Infect Control. 2016 Nov;44(11):1408-10.

6 Lebon D, Biard L, Buyse S, Schnell D, Lengliné E, Roussel C, et al. Gastrointestinal emergencies in critically ill cancer patients. J Crit Care. 2017 Aug;40:69-75.

7 Francisco LM, Sage PT, Sharpe AH. The PD-1 pathway in tolerance and autoimmunity. Immunol Rev. 2010 Jul;236(1):219-42.

8 Reynoso ED, Elpek KG, Francisco L, Bronson R, Bellemare-Pelletier A, Sharpe AH, et al. Intestinal tolerance is converted to autoimmune enteritis upon PD-1 ligand blockade. J Immunol. 2009 Feb;182(4):2102-12.

9 Wang PF, Chen Y, Song SY, Wang TJ, Ji WJ, Li SW, et al. Immune-related adverse events associated with antiPD-1/PD-L1 treatment for malignancies: A meta-analysis. Front Pharmacol. 2017 Oct;8:730.

10 Wang DY, Salem JE, Cohen JV, Chandra S, Menzer C, Ye F, et al. Fatal toxic effects associated with immune checkpoint inhibitors: A systematic review and meta-analysis. JAMA Oncol. 2018 Dec;4(12):1721-8.

11 Celli R, Kluger HM, Zhang X. Anti-PD-1 therapy-associated perforating colitis. Case Rep Gastrointest Med. 2018 May;2018:3406437.

12 Gupta A, Khanna S. Ipilimumab-associated colitis or refractory Clostridium difficile infection? BMJ Case Rep. 2015 Jul;2015:bcr2015211160.

13 Zhang T, Lin QY, Fei JX, Zhang Y, Lin MY, Jiang SH, et al. Clostridium difficile infection worsen outcome of hospitalized patients with inflammatory bowel disease. Sci Rep. 2016 Jul;6(1):29791. 


\section{Case Reports in Oncology}

Case Rep Oncol 2019;12:164-170

DOI: $10.1159 / 000497155$

(C) 2019 The Author(s). Published by S. Karger AG, Basel www.karger.com/cro

Zhou et al.: Pembrolizumab-Induced Immune-Mediated Colitis in a Patient with

Concurrent Clostridium Difficile Infection
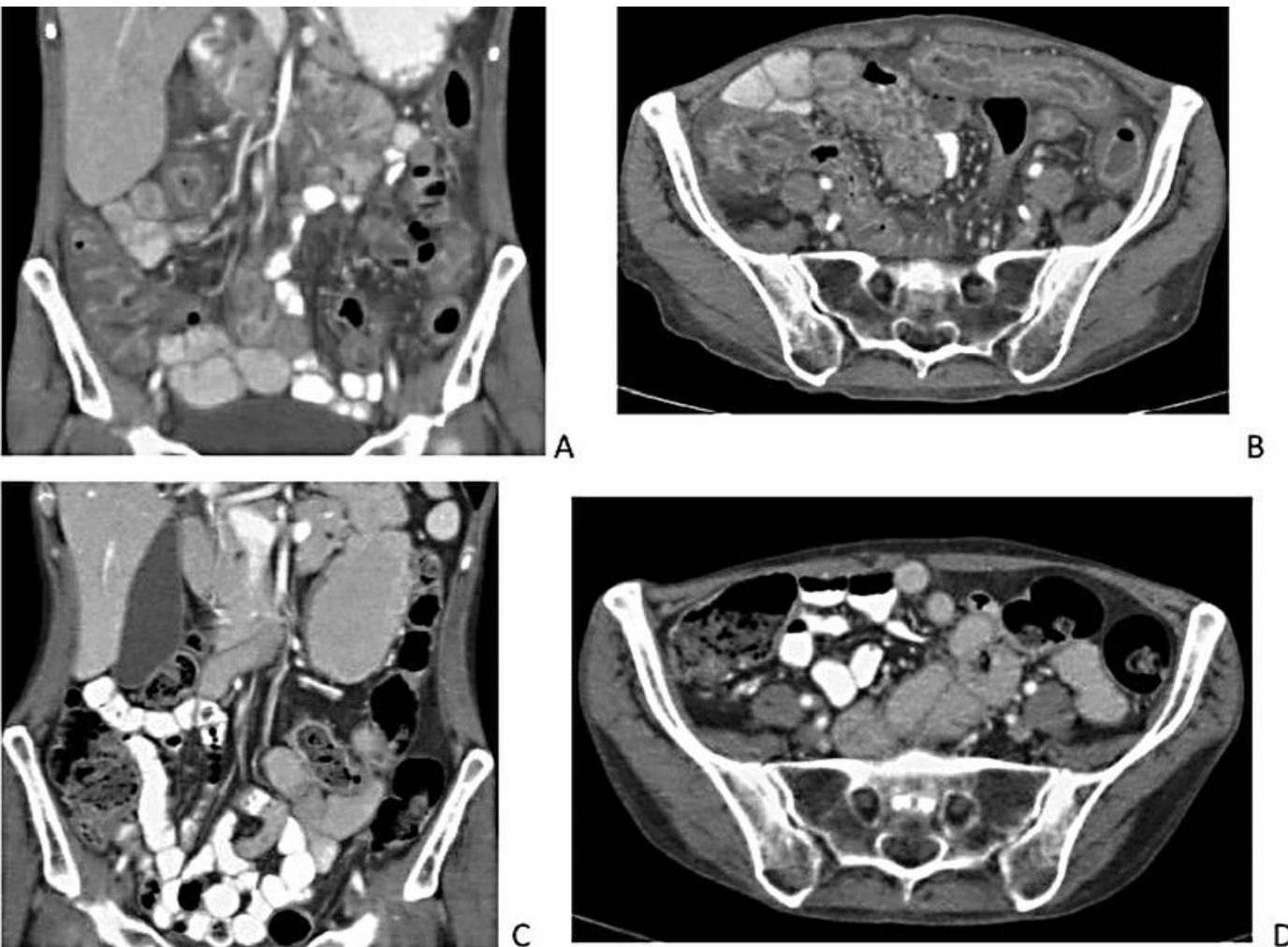

Fig. 1. Abdominal CT scans before initiation of corticosteroids and 30 days into taper course. (A) and (B) Pan-colitis and terminal ileitis on abdominal CT on Hospital Day 3 before Initiation of methylprednisolone. (C) and (D) Complete resolution of colitis and ileitis 30 days into oral prednisone taper.

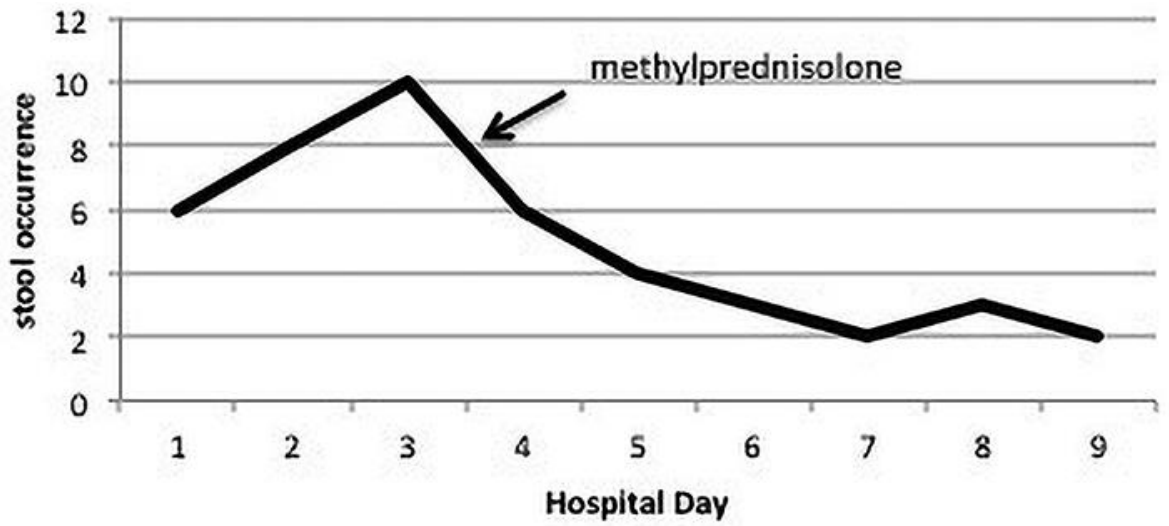

Fig. 2. Number of stool occurrences throughout the hospital course. 
Zhou et al.: Pembrolizumab-Induced Immune-Mediated Colitis in a Patient with Concurrent Clostridium Difficile Infection

Table 1. Results of laboratory testing on Day 1, Day 5 and Day 9 of hospitalization

\begin{tabular}{llllc}
\hline & Day 1 & Day 5 & Day 9 & Reference range \\
\hline $\begin{array}{l}\text { Complete blood count } \\
\text { White blood cell count, } \times 10^{9} / \mathrm{L}\end{array}$ & 2.4 & 1.9 & 7.3 & $4.5-11.5$ \\
$\quad$ Neutrophils, \% & 51.1 & 4.0 & 40.0 & $31.0-76.0$ \\
Lymphocytes, \% & 47.7 & 89.0 & 40.0 & $24.0-44.0$ \\
Monocytes, \% & 0.4 & 7.0 & 15.0 & $2.0-11.0$ \\
Absolute neutrophil count, $\times 10^{9} / \mathrm{L}$ & 1.23 & 0.08 & 3.07 & $1.50-8.00$ \\
Hemoglobin, g/dL & 9.3 & 8.8 & 8.5 & $12.0-15.0$ \\
Hematocrit, \% & 30.0 & 27.2 & 26.8 & $36.0-46.0$ \\
Platelet count, $\times 10^{9} / \mathrm{L}$ & 285 & 20 & 42 & $150-400$ \\
Basic metabolic panel & & & & \\
Glucose, mg/dL & 127 & 92 & 95 & $68-110$ \\
Sodium, mmol/L & 135 & 135 & 140 & $135-148$ \\
Potassium, mmol/L & 3.1 & 3.3 & 3.5 & $3.3-5.3$ \\
Bicarbonate, mmol/L & 30 & 24 & 29 & $21-30$ \\
Chloride, mmol/L & 93 & 105 & 105 & $97-111$ \\
Blood urea nitrogen, mg/dL & 3 & $<3$ & 7 & $8-22$ \\
Creatinine, mg/dL & 0.74 & 0.58 & 0.71 & $0.70-1.50$ \\
Calcium, mg/dL & 8.2 & 7.5 & 8.2 & $8.4-10.4$ \\
\hline
\end{tabular}

Stool C. diff DNA was positive 7 days prior to hospitalization. Repeat Stool C. diff DNA on Hospital Day 4 was negative. On Hospital Day 4, stool was also negative for bacteria, ova and parasites as well as Giardia and Cryptosporidium antigens. 